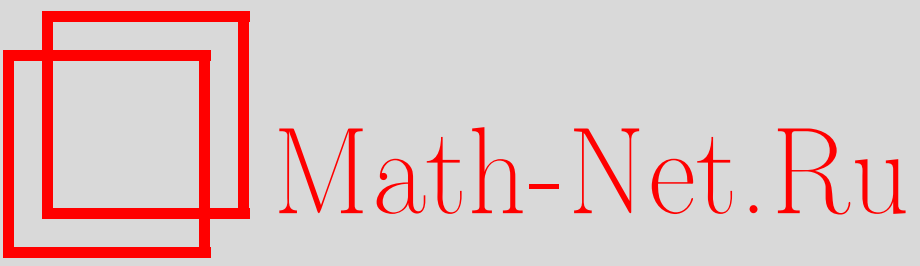

А. А. Гайфуллин, Нервы групп Кокстера, УМН, 2003, том 58, выпуск 3, 189-190

DOI: https://doi.org/10.4213/rm633

Использование Общероссийского математического портала Math-Net.Ru подразумевает, что вы прочитали и согласны с пользовательским соглашением

http: //www.mathnet.ru/rus/agreement

Параметры загрузки:

IP : 3.82 .47 .9

26 апреля 2023 г., $17: 59: 14$ 


\title{
НЕРВЫ ГРУПП КОКСТЕРА
}

\author{
А. А. ГАЙфУллин
}

Cистемой Кокстера назьвается пара $(W, S)$, где $S=\left\{s_{1}, s_{2}, \ldots, s_{m}\right\}$ - конечное множество, $W$ - группа, задаваемая системой образующих $S$ и соотношениями $s_{i}^{2}=1, i=1, \ldots, m$, $\left(s_{i} s_{j}\right)^{n_{i j}}=1, i, j=1, \ldots, m, i \neq j$, где $n_{i j} \in\{2,3, \ldots, \infty\}, n_{i j}=n_{j i}\left(n_{i j}=\infty\right.$ означает отсутствие соответствующего соотношения). Группа $W$, имеющая такую систему образующих, называется группой Kокстера. Система Кокстера $(W, S)$ называется конечной, если группа $W$ конечна. Для любого подмножества $B \subset S$ обозначим через $W_{B}$ подгруппу группы $W$, порожденную образующими из множества $B$.

Нерв ом системы Кокстера $(W, S)$ назьвается симплициальньй комплекс $N(W, S)$ на множестве вершин $S$, симплексы которого задаются множествами $\sigma$ такими, что группа $W_{\sigma}$ конечна.

Целюю этой статьи является описание систем Кокстера, нервы которых являются псевдомногообразиями или горенштейновыми ${ }^{*}$ комплексами и одновременно обладают довольно высокой степенью смежностности (определения см. ниже, а детали в [1]).

Симплициальньй комплекс $L$ размерности $n$ назьвается горенитейновым ${ }^{*}$, если $H_{*}(\operatorname{Lk} \sigma)$ $=H_{*}\left(S^{n-\operatorname{dim} \sigma-1}\right)$ для любого $\sigma \in L$ (мы полагаем, что Lk $\varnothing=L$ и $\operatorname{dim} \varnothing=-1$ ). Симплициальный комплекс $L$ называется $k$-смежнностным, если любое подмножество из не более чем $k$ его вершин задает симплекс этого комплекса. Графом Kокстера системы Кокстера $(W, S)$ называется граф $\Gamma(W, S)$ на множестве вершин $S$, в котором вершины $s_{i}$ и $s_{j}(i \neq j)$ соединены $\left(n_{i j}-2\right)$ ребрами (в граф̆е допускаются кратные ребра, но не может быть ребер, у которых совпадают начало и конец).

Теорема 1. Нерв системы Кокстера $(W, S)$ является 5-смежсностным псевдомногообразием тогда и только тогда, когда система $(W, S)$ представляется в виде прямо20 произведения конечного числа систем Кокстера таких, что граф Кокстера каждой из них имеет не менее 6 вершин и совпадает с одним из графов, изображенных на puc. $1, \mathrm{a}-\mathrm{x}$.

ТеОрема 2. Нерв системы Кокстера $(W, S)$ является 4-смежностным горенштейновым * комплексом тогда и только тогда, когда система $(W, S)$ представляется в виде прямого произведения конечного числа систем Кокстера таких, что граф Кокстера каждой из них имеет не менее 5 вершин и совпадает с одним из графов, изображенных на рис. 1.

ЗАмечаниЕ. Нерв системы Кокстера $(W, S)$ является 9-смежностным комплексом тогда и только тогда, когда система $(W, S)$ представляется в виде прямого произведения конечного числа систем Кокстера таких, что графф Кокстера каждой из них либо имеет не менее 10 вершин и изоморфен одному из графов, изображенных на рис. 1, а-г, либо изоморфен графу Кокстера конечной системы Кокстера.

Список всех связных графов, являющихся графами Кокстера конечных систем Кокстера, приведен в [2]. Система Кокстера конечна тогда и толшко тогда, когда ее граф̆ Кокстера является несвязньм объединением графов из этого списка. Графы, изображенные на рис. $1, \mathrm{a}-0,-$ в точности все связные графы, имеющие не менее 5 вершин, не изоморфоные графам Кокстера конечных систем Кокстера, но такие, что все их собственные полные подграфы изоморфыны графам Кокстера конечных систем Кокстера. Поэтому нервы систем Кокстера с графрами Кокстера, изображенными на рис. $1, \mathrm{a}-0$, изоморффны границам симплексов. Легко проверить, что нерв системы Кокстера с графом Кокстера, изображенньм на рис. 1 , п, изоморфен комплексу $\partial \Delta^{4} * \partial \Delta^{4}$.

Пусть $N$ - нерв системы Кокстера с графом Кокстера, изображенным на рис. 1, р. Тогда $N$ - 4-смежностньй комплекс, не являющийся соединением нескольких границ симплексов, но изоморфньй границе 9-мерного выпуклого симплициального многогранника с 12 вершинами. Например, можно взять многогранник с вершинами $(1,0,0,0,0,0,0,0,0),(0,1,0,0,0,0,0,0,0), \ldots$, $(0,0,0,0,0,0,0,0,1),(2,2,5,4,5,9,3,4,4),(3,4,4,2,2,5,4,5,9),(4,5,9,3,4,4,2,2,5)$.

Нерв прямого произведения систем Кокстера изоморфен соединению их нервов. Таким образом, из теорем 1 и 2 получаем: 
СлеДСТВИЕ 1. 5-смежнностное псевдомногообразие является нервом какой-нибудь системы Кокстера тогда и только тогда, когда оно является соединением нескольких гранич симплексов.

СледствиЕ 2. 4-смежностный горенштейнов* комплекс является нервом некоторой системы Кокстера тогда и только тогда, когда он является соединением нескольких комплексов, каждый из которых изоморфен либо границе симплекса, либо комплексу N. В частности, любой такой комплекс изоморфен границе выпуклого симпличиального многогранника.

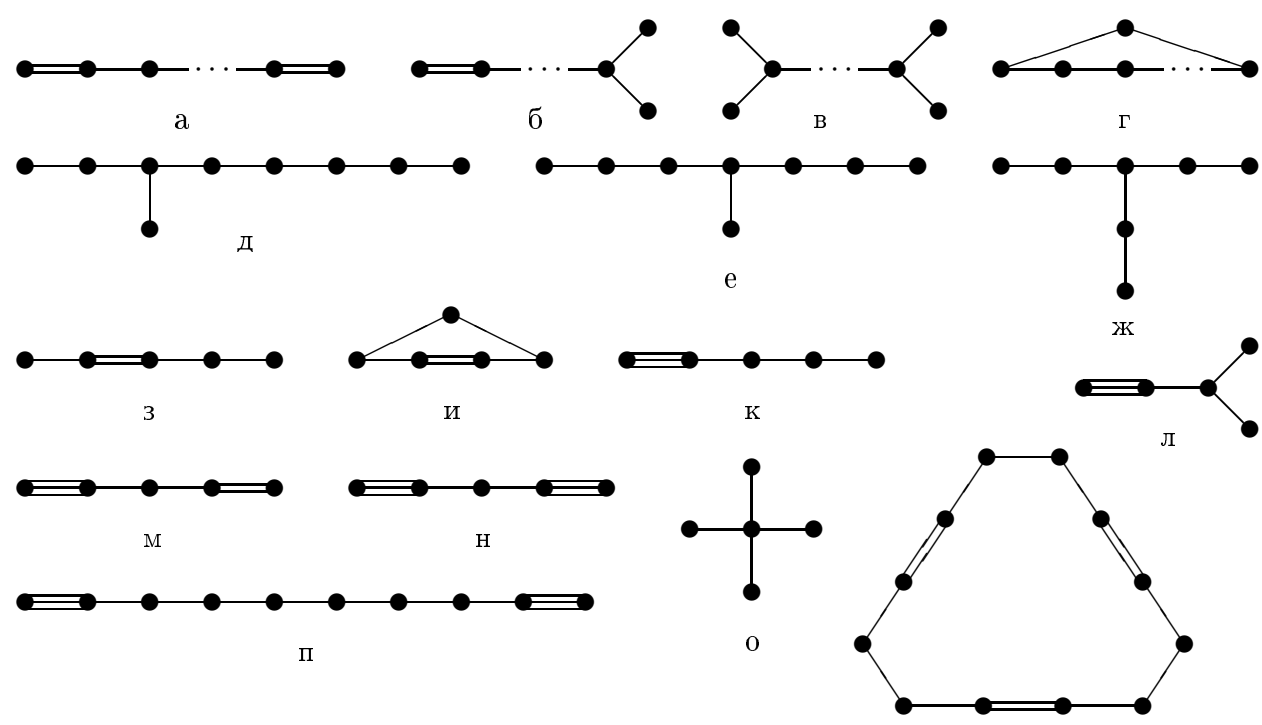

$\mathrm{p}$

Пусть система Кокстера $(W, S)$ является прямым произведением систем Кокстера с графами Кокстера, изображенными на рис. 1 . Тогда ее граф̆ Кокстера $\Gamma(W, S)$ - несвязное объединение графов, изображенных на рис. 1 . Обозначим через $k_{j}(W, S)$ количество компонент связности граффа $\Gamma(W, S)$, имеющих ровно $j$ вершин и изоморфных одному из графов на рис. 1, а-о, через $k_{10}^{*}(W, S)$ и $k_{12}^{*}(W, S)$ - количества компонент связности графа $\Gamma(W, S)$, изоморфных граффам на рис. 1, п, и на рис. $1, \mathrm{p}$, соответственно.

Теорема 3. Две системы Кокстера $(W, S)$ u $\left(W^{\prime}, S^{\prime}\right)$, каждая из которых является прямым произведением нескольких систем Кокстера с графами Кокстера, изображенными на рис. 1, имеют изоморфнье нервы тогда и только тогда, когда $k_{j}(W, S)=k_{j}\left(W^{\prime}, S^{\prime}\right), j \geqslant 6, k_{5}(W, S)+2 k_{10}^{*}(W, S)=k_{5}\left(W^{\prime}, S^{\prime}\right)+2 k_{10}^{*}\left(W^{\prime}, S^{\prime}\right) u$ $k_{12}^{*}(W, S)=k_{12}^{*}\left(W^{\prime}, S^{\prime}\right)$.

Я благодарен моему научному руководителю В. М. Бухштаберу за постоянное внимание к моей работе, а также Н.Э. Добринской, Т. Е. Панову и Г. И. Шарыгину за полезные обсуждения.

\section{СПИСОК ЛИТЕРАТУРЫ}

[1] В. М. Бухштабер, Т. Е. Панов // УМН. 2000. Т. 55. № 5. С. 3-106. [2] Н. Бурбаки. Группы и алгебры Ли. М.: Мир, 1972.

Московский государственньй

Принято редколлегией университет им. М.В. Ломоносова 24.03 .2003 